OPEN ACCESS

Edited by:

Yuri Shavrukov,

Flinders University, Australia

Reviewed by:

Farid Shekari,

University of Zanjan, Iran

Rudo Ngara,

University of the Free State

South Africa

*Correspondence:

Xiaodong Wang

zhbwxd@hebau.edu.cn

Mingming Yang

myang@nwsuaf.edu.cn

Wenchao Zhen

wenchao@hebau.edu.cn

${ }^{\dagger}$ These authors have contributed equally to this work

Specialty section: This article was submitted to

Plant Abiotic Stress,

a section of the journal

Frontiers in Plant Science

Received: 28 November 2021

Accepted: 18 January 2022

Published: 24 February 2022

Citation:

Liu X, Wang X, Liu P, Bao X, Hou X, Yang $M$ and Zhen W (2022) Rehydration Compensation of Winter

Wheat Is Mediated by Hormone Metabolism and De-Peroxidative Activities Under Field Conditions.

Front. Plant Sci. 13:823846. doi: 10.3389/fpls.2022.823846

\section{Rehydration Compensation of Winter Wheat Is Mediated by Hormone Metabolism and De-Peroxidative Activities Under Field Conditions}

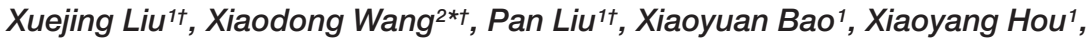 \\ Mingming Yang ${ }^{3 *}$ and Wenchao Zhen ${ }^{1 *}$

\begin{abstract}
1 State Key Laboratory of North China Crop Improvement and Regulation, Key Laboratory of Crop Growth Regulation of Hebei Province, College of Agronomy, Hebei Agricultural University, Baoding, China, ${ }^{2}$ College of Plant Protection, Hebei
\end{abstract} \\ Agricultural University, Baoding, China, ${ }^{3}$ College of Agronomy, Northwest A\&F University, Xianyang, China
}

Water deficit and rehydration frequently occur during wheat cultivation. Previous investigations focused on the water deficit and many drought-responsive genes have been identified in winter wheat. However, the hormone-related metabolic responses and de-peroxidative activities associated with rehydration are largely unknown. In this study, leaves of two winter wheat cultivars, "Hengguan35" (HG, drought-tolerant cultivar) and "Shinong086" (SN, drought-sensitive cultivar), were used to investigate water deficit and the post-rehydration process. Rehydration significantly promoted wheat growth and postponed spike development. Quantifications of antioxidant enzymes, osmotic stress-related substances, and phytohormones revealed that rehydration alleviated the peroxidation and osmotic stress caused by water deficit in both cultivars. The wheat cultivar HG showed a better rehydration-compensation phenotype than SN. Phytohormones, including abscisic acid, gibberellin (GA), jasmonic acid (JA), and salicylic acid (SA), were detected using high-performance liquid chromatography and shown to be responsible for the rehydration process. A transcriptome analysis showed that differentially expressed genes related to rehydration were enriched in hormone metabolism- and de-peroxidative stress-related pathways. Suppression of genes associated with abscisic acid signaling transduction were much stronger in HG than in $\mathrm{SN}$ upon rehydration treatment. $\mathrm{HG}$ also kept a more balanced expression of genes involved in reactive oxygen species pathway than SN. In conclusion, we clarified the hormonal changes and transcriptional profiles of drought-resistant and -sensitive winter wheat cultivars in response to drought and rehydration, and we provided insights into the molecular processes involved in rehydration compensation.

Keywords: wheat, rehydration compensation, drought stress, hormone metabolism, transcriptomic, deperoxidative stress 


\section{INTRODUCTION}

Wheat is an important global staple crop vulnerable to climate conditions and its yield is associated with water supply during the cultivation season (Araya et al., 2019; Wang et al., 2021). Winter wheat relies on over-extracted groundwater to maintain a high yield, which causes severe ecological problems, including large-scale soil surface subsidence (Kong et al., 2016). Therefore, winter wheat production exacerbates water resource shortages, which is becoming increasingly serious (Huang et al., 2019). Rehydration compensation is a common phenomenon during crop cultivation. In certain developmental periods of crops, water limited irrigation triggers a compensatory promotion of growth after release of the drought stress, which simultaneously improves water use efficiency, yield, and quality of crops (Araya et al., 2019). An efficient rehydration compensation is dependent on a good balancing between "self-protection" of crops upon drought stress and "compensation" after rehydration treatment (Zhao et al., 2020). Consequently, it is crucial to clarify the physiological changes and molecular mechanisms associated with both water deficit and rehydration in wheat (Lopes et al., 2012; Song et al., 2017).

Morphological adaptations of crops are common features of rehydration compensation (Ahmad et al., 2015; Chen et al., 2020; Khadka et al., 2020). Compared with normal-watered plants, rehydration treatment at the re-greening stage of wheat can even elevate plant height, ear length, and flag leaf area (Baczek-Kwinta et al., 2006; Trujillo et al., 2013; Caldeira et al., 2014; Husen et al., 2014; Wang et al., 2017). Plant antioxidant metabolism is closely associated with water deficit and rehydration (Baczek-Kwinta et al., 2006; Furlan et al., 2016). Antioxidant enzymes are maintained at higher levels to balance the free radicals in plants to avoid damage from peroxidation under drought-stress conditions. During drought stress and rehydration, crops maintain a dynamic balance between the production and elimination of free radicals of ROS by regulating genes encoding antioxidant enzymes (He et al., 2012; Gupta et al., 2020), which has been evidenced by a series of studies in cotton, maize, and other crops (Koffler et al., 2014; Ashraf et al., 2015; Yi et al., 2016). For example, the activities of antioxidant enzymes superoxide dismutase (SOD) and peroxidase (POD) in maize leaves increased slowly upon drought stress but decreased dramatically after rehydration (Zheng et al., 2010; Ye et al., 2016). The degree of rehydration compensation is dependent on the tolerance of plant to drought stress (Zhang et al., 2018; Li X. R. et al., 2020). The association between osmotic stress regulatory ability and rehydration compensation have been evaluated in soybean and maize (Blum, 2017; Du et al., 2020). Genes regulating the production of proline (Pro), including pyrroline-5-carboxylate synthetase, harpin-encoding protein, and EF-Hand family protein, participate in plant tolerance to drought stress (Wang et al., 2013; Qiu et al., 2020; Anwar et al., 2021). The Arabidopsis C-repeat-binding factor gene (AtCBF4) is another key component in producing soluble sugars and enhancing the drought tolerance of plants (Zhang Z. et al., 2020). Water deficit triggers complex signal transduction pathways involved in hormone metabolism. The phytohormone changes result in plant physiological, ecological, and morphological responses against abiotic stresses. Upon drought stress, abscisic acid (ABA) accumulated in plant leaves and suppressed stomatal conduction, which eventually reduced the transpiration rate and prevented excessive water loss (Mega et al., 2019). The Oryza sativa Abscisic Stressripening 5 (OsASR5) gene plays an essential role in responses to drought stress by regulating ABA biosynthesis, promoting stomatal closure, and preventing drought stress-related protein inactivation (Li et al., 2017). Sajjad et al. (2021) found that a knock-out of cotton (Gossypium hirsutum) WUSCHEL-like homeobox 4 (GhWOX4) gene results in severely impaired vascular growth and drought tolerance. Several phytohormones, including auxin (AUX), indoleacetic acid (IAA), ABA and ethylene, are significantly induced in Arabidopsis GhWOX4overexpression lines (Sajjad et al., 2021). During the rehydration, phytohormones also play crucial roles in the recovery of plant growth and long-term compensation (Truskina et al., 2021). To date, investigations have focused on the identification and characterization of drought-responsive genes in winter wheat, but little is known about the regulatory mechanisms of rehydration compensation. This study explored the hormonal changes and transcriptional profiles of drought-resistant and sensitive winter wheat cultivars in response to rehydration. The physiological responses and molecular mechanisms of wheat plants during rehydration compensation were clarified.

\section{MATERIALS AND METHODS}

\section{Plant Material, Growth Conditions, Drought Stress, and Rehydration Treatment}

Drought-tolerant variety Hengguan35 (HG, sourced from Drought Agricultural Experiment Station of Hebei Academy of Agriculture and Forestry Sciences, Hebei, China) and droughtsensitive variety Shinong086 (SN, sourced from Hebei Dadi Seed Industry Co., Ltd., Hebei, China) were used in this study (Li D. et al., 2020). The seeds were sown in a field at the Shenzhou Experimental Station of Arid Crops Research Institute, Hebei Academy of Agriculture and Forestry Sciences (Hebei, China, $37.91^{\circ} \mathrm{N}, 115.71^{\circ} \mathrm{E}$ ) on October 13, 2018. All winter wheat plants were grown under rain-proof shelters to prevent watering from the rain (Figure 1A). The area of each plot was $40 \mathrm{~m}^{2}(10 \mathrm{~m} \times 4$ m). On November $28,2018,3 \mathrm{~m}^{3}$ water was irrigated for each plot, as to ensure safety of overwinter. All wheat plants were subjected to continuous drought stress until the fourth-leaf stage in spring of 2019. A Trimer Pico 64 portable soil moisture meter (TDR, IMIKO, Germany) was used to measure the $0-200 \mathrm{~cm}$ layer soil moisture content $(\mathrm{v} / \mathrm{v})$ at every $20 \mathrm{~cm}$ soil depth. At the four-leaf stage of wheat plant [168 days after seed sowing (DAS)], and the soil water content of 0-60 $\mathrm{cm}$ layer was about $20 \%$, two treatments were set up (Figure 1B). For Treatment 1 (rehydrated treatment), irrigation was applied, each plot was supplemented with $2.8 \mathrm{~m}^{3}$ water for irrigation until the water 
A

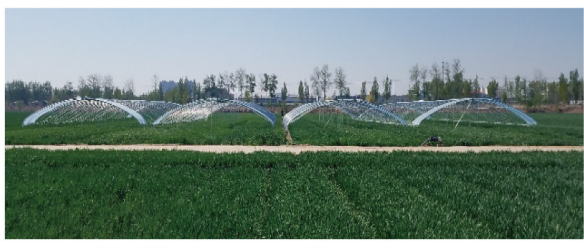

Folded state of canopy top

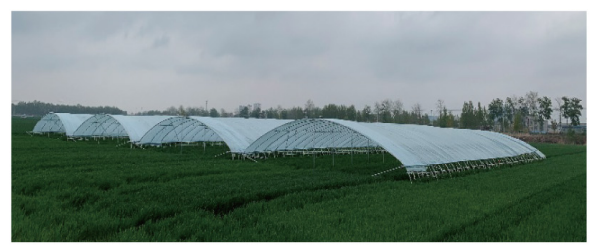

Unfolded state of canopy top

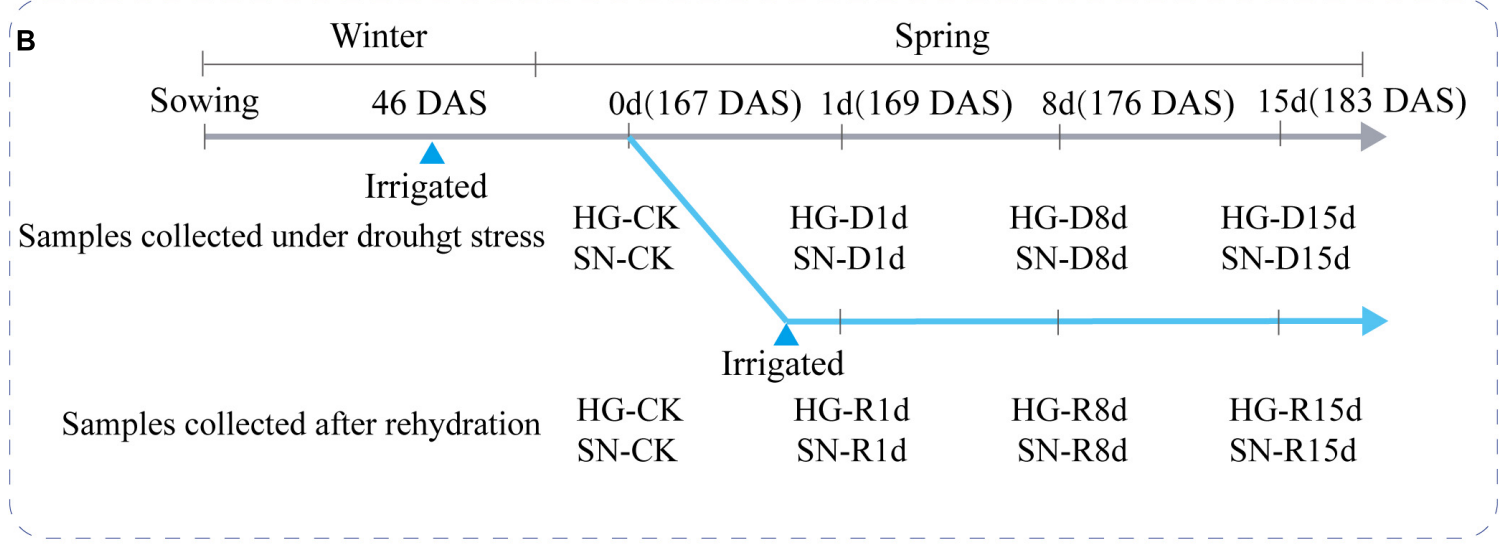

C

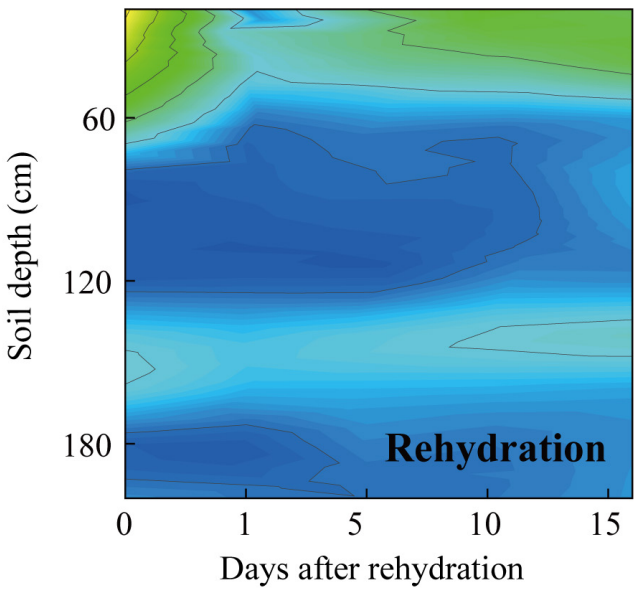

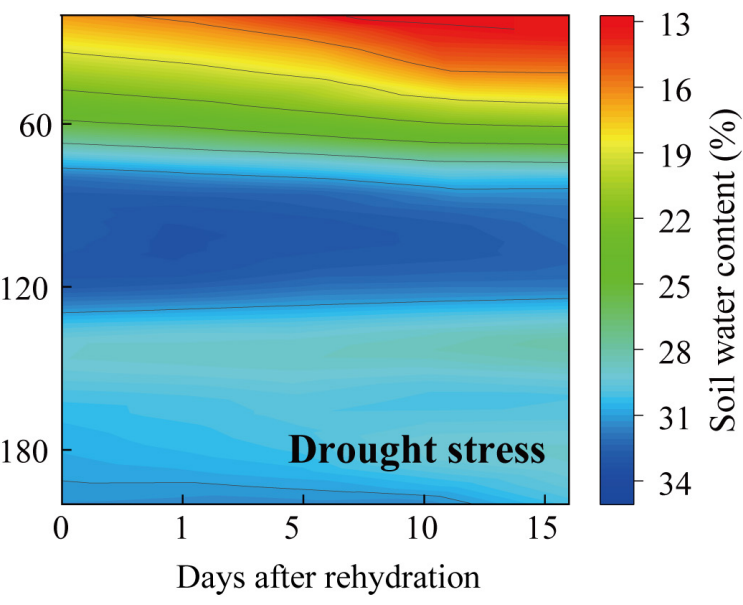

FIGURE 1 | Schematic representation of rehydration experimental design. (A) Rain shelter for field experiments. (B) Timeline for rehydration treatment and sample collection. (C) Profile of the relative soil-water content (RSWC) during different rehydration stages. Scale bar represents soil moisture content in percentage. R, rehydrated plants; D, plants under continuous drought stress.

content of the $0-60 \mathrm{~cm}$ soil layer reached $80 \%$ of the field waterholding capacity. For Treatment 2 (continuous drought stress treatment), no irrigation was applied. Each treatment includes three plots. Wheat leaf samples were collected in triplicate from both rehydrated and continuous-drought-stressed seedlings at 1 (169 DAS), 8 (176 DAS), 15 (183 DAS) days after irrigation. Leaf samples collected 1 day before the rehydration served as control [167 DAS, 0 days post-rehydration (dpr)]. The soil water content of 0-60 cm layer in the rehydrated field was greater than $25 \%$ at $15 \mathrm{dpr}$, indicating a sufficient water supply for wheat growth. Soil water content of 0-60 cm layer in the continuous-droughtstressed field was consistently lower than 20\% (Figure 1C).
Plant height was determined by measuring the main tiller at 15 dpr (183 days after sowing). To monitor the development of the wheat embryos, developing spikes were collected at 1, 8, and $15 \mathrm{dpr}$ with awns and rachis removed. The isolated embryos were observed using anatomic microscope. For evaluation of physiological activities, quantification of phytohormone content, RNA sequencing, and qRT-PCR assay, samples were collected from the fully expanded fourth leaves of wheat plants at 1,8 , and $15 \mathrm{dpr}$ (Figure 1B). Leaf samples from 10 independent plants were combined as one biological replicate. Three biological replicates were collected at each time point. All the samples were immediately frozen in liquid nitrogen and stored at $-80^{\circ} \mathrm{C}$. 


\section{Physiological Traits Measurement}

The SOD activity was measured using the nitro-blue tetrazolium photoreduction method (Giannopolitis and Ries, 1977). The POD activity was measured using the guaiacol method (Han et al., 2008). The CAT activity was estimated by measuring the initial disappearance rate of $\mathrm{H}_{2} \mathrm{O}_{2}$ (Shimizu, 1987). The APX activity was measured using the method from Asada (1980). The MDA content was determined using the thiobarbituric acid reaction as described previously (Bramlage, 1992). The soluble sugars content was evaluated using the anthrone colorimetric method (Miller, 1959). The proline content was quantified using the ninhydrin colorimetric method (Bates et al., 1973). The soluble protein content was measured using the Coomassie brilliant blue method (Campion et al., 2011).

\section{Phytohormone Content Quantification}

Five endogenous phytohormones in wheat leaves, including $\mathrm{ABA}, \mathrm{IAA}, \mathrm{GA}, \mathrm{SA}$, and JA, were quantified using the highperformance liquid chromatography (HPLC) method. Briefly, $2 \mathrm{~g}$ fresh wheat leaves were weighed and ground into a slurry with $10 \mathrm{ml}$ pre-cooled $80 \%$ methanol in an ice bath. This slurry was sealed in plastic wrap and kept overnight at $4^{\circ} \mathrm{C}$. The supernatant was obtained by centrifugation at $8,000 \mathrm{~g}$ at $4^{\circ} \mathrm{C}$ for $10 \mathrm{~min}$. The residue was re-suspended in $8 \mathrm{ml}$ pre-cooled $80 \%$ methanol, and the supernatant was collected again by centrifugation. The filtrate was concentrated under reduced pressure at $40^{\circ} \mathrm{C}$ to one-third of the original volume. Samples were extracted and decolorized using $30 \mathrm{ml}$ petroleum ether three times, with the ether phase discarded each time. The aqueous phase was extracted using $20 \mathrm{ml}$ ethyl acetate three times, and the ester phases were combined. Samples were then dried by evaporation under reduced pressure at $40^{\circ} \mathrm{C}$. Samples were mixed with $2 \mathrm{ml}$ acetic acid solution ( $\mathrm{pH}$ 3.5), purified using a Sep-Pak C18 cartridge, eluted with methanol, and concentrated to dry powders under reduced pressure at $40^{\circ} \mathrm{C}$. Before high-performance liquid chromatography (HPLC), samples were dissolved, diluted to a total volume of $2 \mathrm{ml}$ in a mobile phase, and filtered through a 0.45 $\mu \mathrm{m}$ microporous membrane. An Agilent 1260 High Performance Liquid Chromatograph was employed. The chromatographic column used was an Eclipse XDB-C18 $(250 \mathrm{~mm} \times 4.6 \mathrm{~mm}$, 5 $\mu \mathrm{m}$, Agilent, CA, United States). Mobile phase A was methanol, and phase $\mathrm{B}$ was an aqueous acetic acid solution ( $\mathrm{pH}$ 3.5). Five endogenous phytohormones in wheat leaves were separated at the same time by gradient elution. The gradient conditions were set as 0-7 $\mathrm{min}, 20 \% \mathrm{~A} ; 7-10 \mathrm{~min}, 20-28 \% \mathrm{~A} ; 0-17 \mathrm{~min}, 28 \% \mathrm{~A} ; 17-$ $19 \mathrm{~min}, 28-40 \% \mathrm{~A}$; and $19-35 \mathrm{~min}, 40 \% \mathrm{~A}$. The flow rate was $1 \mathrm{~mL} / \mathrm{min}$, and the injection volume was $10 \mu \mathrm{L}$. The detector wavelengths were set to 254 and $240 \mathrm{~nm}$, and the detection temperature was room temperature (Zhong et al., 2013).

\section{RNA-Seq and Bioinformatics Analysis}

Total RNA was extracted using TRIzol reagent (Invitrogen, Thermo Fisher Scientific, United States) in accordance with the manufacturer's instructions. Sequencing libraries were generated using NEBNext ${ }^{\circledR}$ Ultra $^{\text {TM }}$ RNA Library Prep Kit for Illumina ${ }^{\circledR}$ (NEB, MA, United Kingdom) following the manufacturer's recommendations. First-strand cDNA was synthesized using a random hexamer primer and M-MuLV Reverse Transcriptase (RNase H-). Second-strand cDNA synthesis was subsequently performed using DNA Polymerase I and RNase H. PCR products were purified (AMPure XP system), and library quality was assessed on the Agilent Bioanalyzer 2100 system. Q20, Q30, and the GC content of the clean data were calculated. Clean reads were aligned to wheat reference genome sequences released by the International Wheat Genome Sequencing Consortium using HISAT2 (Kim et al., 2015).

To assess the variability among samples, we performed a principal component analysis for the overall transcripts of each sample using the prcomp command line with default parameters in the $\mathrm{R}$ software package (Robinson et al., 2010). The comparisons were made between rehydration and control samples at 1,8 , and $15 \mathrm{dpr}$. An adjusted $p$-value ( $q$-value $)<0.05$ and $\mid \log 2$ FoldChange $\mid \geq 1$ were set as the criteria for determining DEGs and classified into clusters using $\mathrm{k}$-means clustering. Heatmaps were visualized using the $\mathrm{R}$ package Complex Heatmap v2.5.5 (Yi et al., 2019). Gene ontology (GO) and Kyoto Encyclopedia of Genes, and Genomes (KEGG) pathway enrichments of DEGs were conducted using the $R$ software package based on the hypergeometric distribution. Raw data for the transcriptome were deposited at NCBI BioProject PRJNA774165.

\section{qRT-PCR}

Total RNA was extracted from samples using a QIAGEN Plant RNA extraction kit (QIAGEN, Hilden, Germany). The first-strand cDNA was synthesized using the EasyScript FirstStrand cDNA reagent (Transgen Biotech, Dalian, China). The wheat Actin gene (GenBank accession AB181991, primers in Supplementary Table 1) was used as an internal reference (Rong et al., 2016). Six genes (wheat genome accessions TraesCS7D02G199900, TraesCS7A02G337000, TraesCS6A02G094400, TraesCS3A02G535300, TraesCS3A02 G081300, and TraesCS2D02G340100) were randomly selected from DEGs for the qRT-PCR assay to evaluate the reliability of the expression levels determined by RNA-seq. Primers for these DEGs were designed (Supplementary Table 1). Three biological replicates were included for each treatment. qRT-PCR was performed using the PerfectStart ${ }^{\mathrm{TM}}$ Green qPCR SuperMix (Transen Biotech, Beijing, China). The threshold values (Ct) were calculated by the Roche LightCycler 96 (Roche, Indianapolis, IN, United States). Gene expression was evaluated using the $2^{-\Delta \Delta \mathrm{Ct}}$ method (Livak and Schmittgen, 2001). The FPKM values of these six DEGs were collected from the transcriptome database to profile the expression patterns. The correlation of overall expression levels of these six genes between qRT-PCR and RNA-seq was calculated using the R software package.

\section{Data Processing and Statistical Analysis}

All of the statistical analyses were performed using SPSS Statistics 22.0 (IBM, NY, United States). Data are presented as means \pm standard error (SE) values. A one-way ANOVA was conducted, and Student's $t$-tests were used to compare treatment means at the $5 \%$ level. 


\section{RESULTS}

\section{A Drought-Tolerant Winter Wheat Cultivar Showed Better \\ Rehydration-Compensation Phenotypes Than a Drought-Susceptible Cultivar}

To investigate whether there is an association between rehydration compensation and drought tolerance, we compared the growth of drought-tolerant cultivar " $\mathrm{HG}$ " and the drought-sensitive cultivar "SN" under rehydration and drought-stress conditions (Figure 1). Plants from both watered (rehydrated, R) and non-watered (continuous drought stress, D) field were collected and photographed at $15 \mathrm{dpr}$ (Figure 2A). The growth of drought-stressed plants of both $\mathrm{HG}$ and $\mathrm{SN}$ were recovered upon rehydration. Plant heights significantly increased after the rehydration treatment, and the drought-tolerant cultivar showed a pronounced faster growth than the drought-sensitive cultivar (Figure 2B). We further checked embryonic development of these wheat plants at 1, 8, $15 \mathrm{dpr}$ (Figure 2C). The ear development was postponed by the rehydration treatment, with much greater delays in $\mathrm{HG}$ than SN. Thus, the drought-tolerant winter wheat cultivar appeared to possess a better rehydration compensation by promoting plant growth and postponing spike development.

\section{Rehydration Alleviated Peroxidation and Osmotic Stress}

To characterize the physiological changes of wheat leaves after being rehydrated, we further measured the SOD, POD, catalase (CAT), and ascorbate peroxidase (APX) activity levels in both HG and SN at $0,1,8$, and $15 \mathrm{dpr}$ (Figure 3). The overall SOD, POD, CAT, and APX activities were suppressed after rehydration, and were much lower in the $\mathrm{HG}$ than $\mathrm{SN}$. We also measured the dynamic changes in MDA, Pro, soluble protein, and soluble sugars levels (Figure 3). Interestingly, all these stress-related contents were reduced after rehydration, and the overall levels of them were lower in HG than those in $\mathrm{SN}$. These results indicated that rehydration process alleviated peroxidative activities and reduced stress-related contents.

\section{Phytohormone Contents of Abscisic Acid, Gibberellin, Jasmonic Acid, and Salicylic Acid Were Reduced Upon Rehydration}

To determine the key phytohormones involved in the rehydration process, we quantified the concentrations of ABA, IAA, GA, JA, and SA using HPLC in both HG and SN leaves at $0,1,8$, and $15 \mathrm{dpr}$ (Figure 4). We observed that the ABA content increased gradually under continuous drought
A

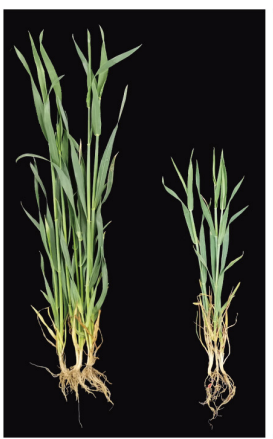

HG-R HG-D

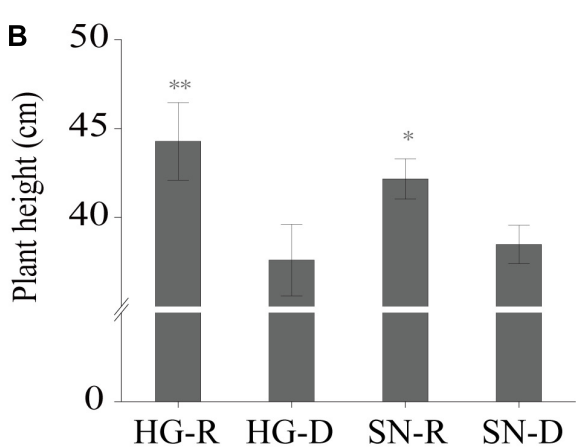

SN-D
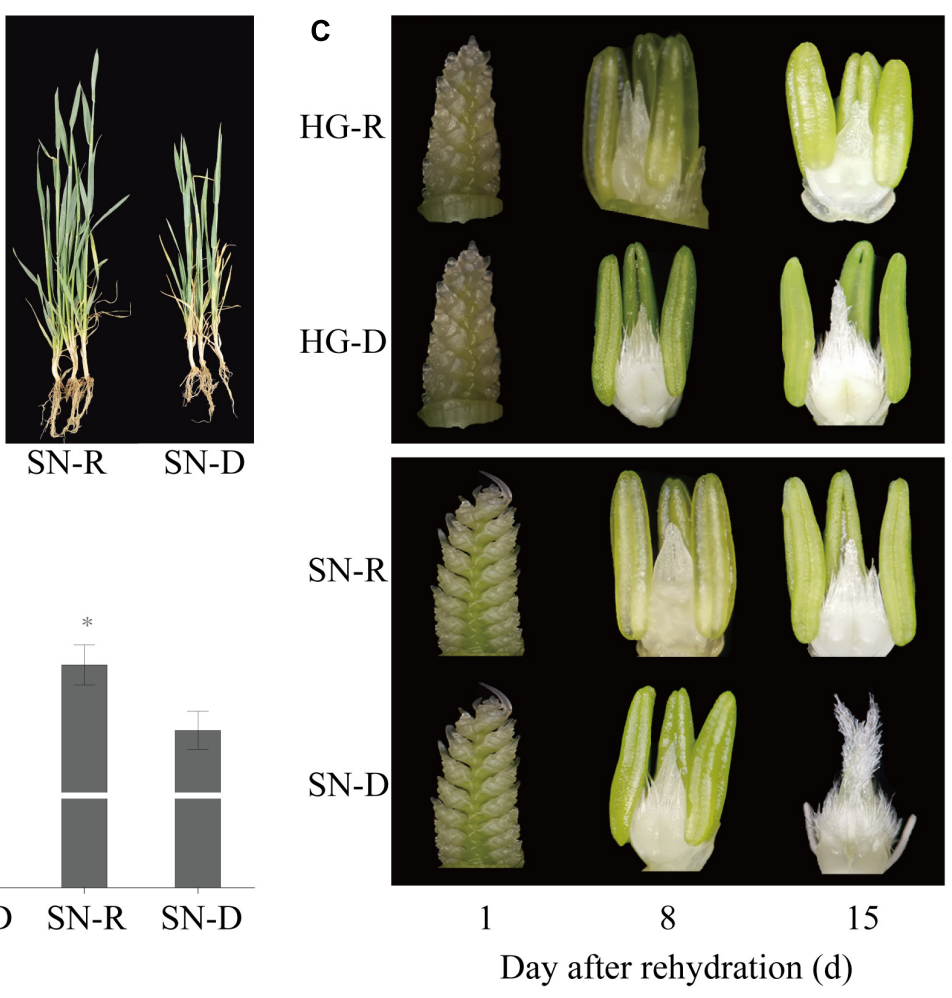

FIGURE 2 | The drought-tolerant wheat variety "Hengguan35" (HG) showed better rehydration-compensation phenotypes than the drought-sensitive wheat variety "Shinong086" (SN). (A) Plant morphology of HG and SN at $15 \mathrm{dpr}$. "*, ${ }^{\star \star}$ " indicate significant difference at 0.05 level. ${ }^{\star \star} P<0.01$; ${ }^{\star} P<0.05$. (B) Plant height was measured at $15 \mathrm{dpr}$. (C) Spikes were isolated and observed using a microscope at 1, 8, and 15 dpr. R, rehydrated plants; D, plants under continuous drought stress. 

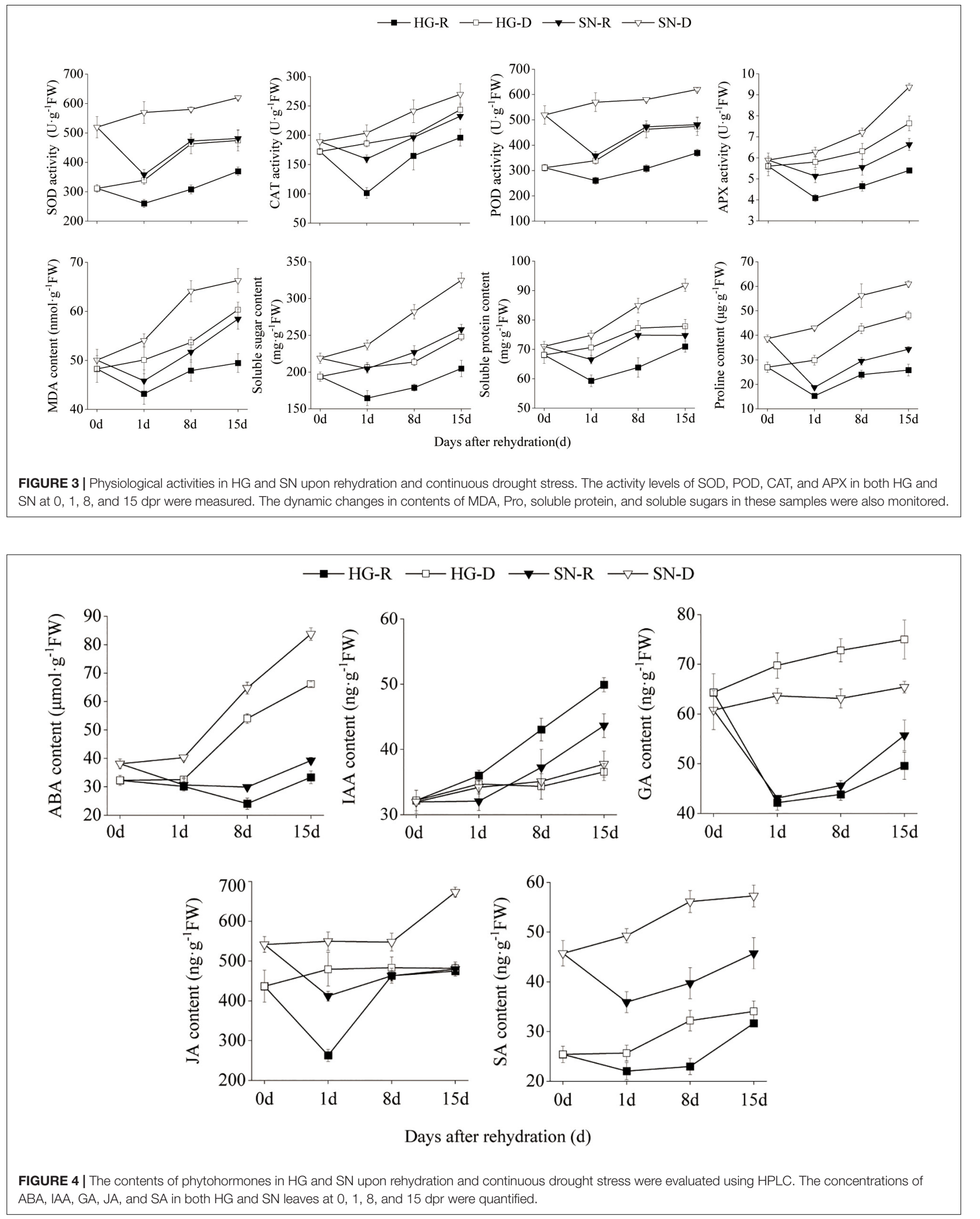
stress, whereas decreased in both SN and HG upon rehydration at 1 and $8 \mathrm{dpr}$. Moreover, the overall ABA content in HG was significantly lower than $\mathrm{SN}$ in either rehydration or drought treatment. The IAA content was accumulated during continuous drought stress, with even higher level in HG than SN. The GA content was reduced in both $\mathrm{HG}$ and $\mathrm{SN}$ upon rehydration. In the continuous drought treatment, the overall GA content in HG was significantly higher than SN. JA content in HG was significantly lower than $\mathrm{SN}$ before the rehydration treatment ( $0 \mathrm{dpr}$ ). The JA content was greatly reduced upon rehydration treatment in both $\mathrm{HG}$ and $\mathrm{SN}$ at $1 \mathrm{dpr}$, and then increased rapidly at $8 \mathrm{dpr}$. The overall SA content in HG was significantly lower than $\mathrm{SN}$ in either rehydration or drought treatment. Upon rehydration treatment, the SA content was decreased in both $\mathrm{HG}$ and $\mathrm{SN}$ at $1 \mathrm{dpr}$, and then increased gradually at 8 and 15 dpr. These results indicated that phytohormone accumulations in $\mathrm{HG}$ and $\mathrm{SN}$ differed before the rehydration treatment, which may result from the drought-tolerant features of these cultivars. The rehydration process was associated with the decreased accumulations of ABA, GA, JA, and SA.

\section{Transcriptome Sequencing Revealed the Molecular Mechanisms of Rehydration}

RNA samples were collected from leaves of $\mathrm{HG}$ and $\mathrm{SN}$ at $0,1,8$, and 15 dpr. Each sample included three biological replicates. In general, 42 RNA samples were prepared and sent for $12-\mathrm{Gb}$ Illumina sequencing (Supplementary Table 2). The Triticum aestivum reference genome (IWGSCV1.1 version) from Ensembl Genomes was used to assemble the transcriptome (Supplementary Table 3). In total, 132,982 genes were mapped to the genome sequence. A principal component analysis revealed a high degree $\left(R^{2}>0.92\right)$ of correlation in the overall gene expression levels among biological replicates (Supplementary Figure 1). Samples were clustered based on genotype (HG or $\mathrm{SN}$ ) and time point $(0,1,8$, or 15 dpr), respectively (Figure 5A). The fragments per kilobase of transcript per million mapped reads (FPKM) value was used to estimate the expression levels of the genes in the transcriptome. The differentially expressed genes (DEGs, q-value $<0.05$, $\mid \log 2$ foldchange $\mid>1$ ) were identified using DESeq2. All the raw data for the transcriptome assembly have been deposited in NCBI under BioProject PRJNA774165.

Compared with the control group before rehydration ( $0 \mathrm{dpr}$ ), we identified 12,073, 13,927, and 14,250 DEGs in rehydrated HG at 1,8 , and $15 \mathrm{dpr}$, respectively. A total of 12,908, 12,079, and 14,169 DEGs were found in rehydrated $\mathrm{SN}$ at 1,8 , and $15 \mathrm{dpr}$, respectively. Compared with samples from $\mathrm{HG}$ at $0 \mathrm{dpr}$, there were 12,592, 6,117 and 11,039 DEGs in the non-watered HG (continuous drought stress) at 1,8 , and $15 \mathrm{dpr}$, respectively. There were 5,224, 8,151 and 11,478 DEGs in the non-watered SN at 1, 8, and $15 \mathrm{dpr}$, respectively. Interestingly, a large number of these

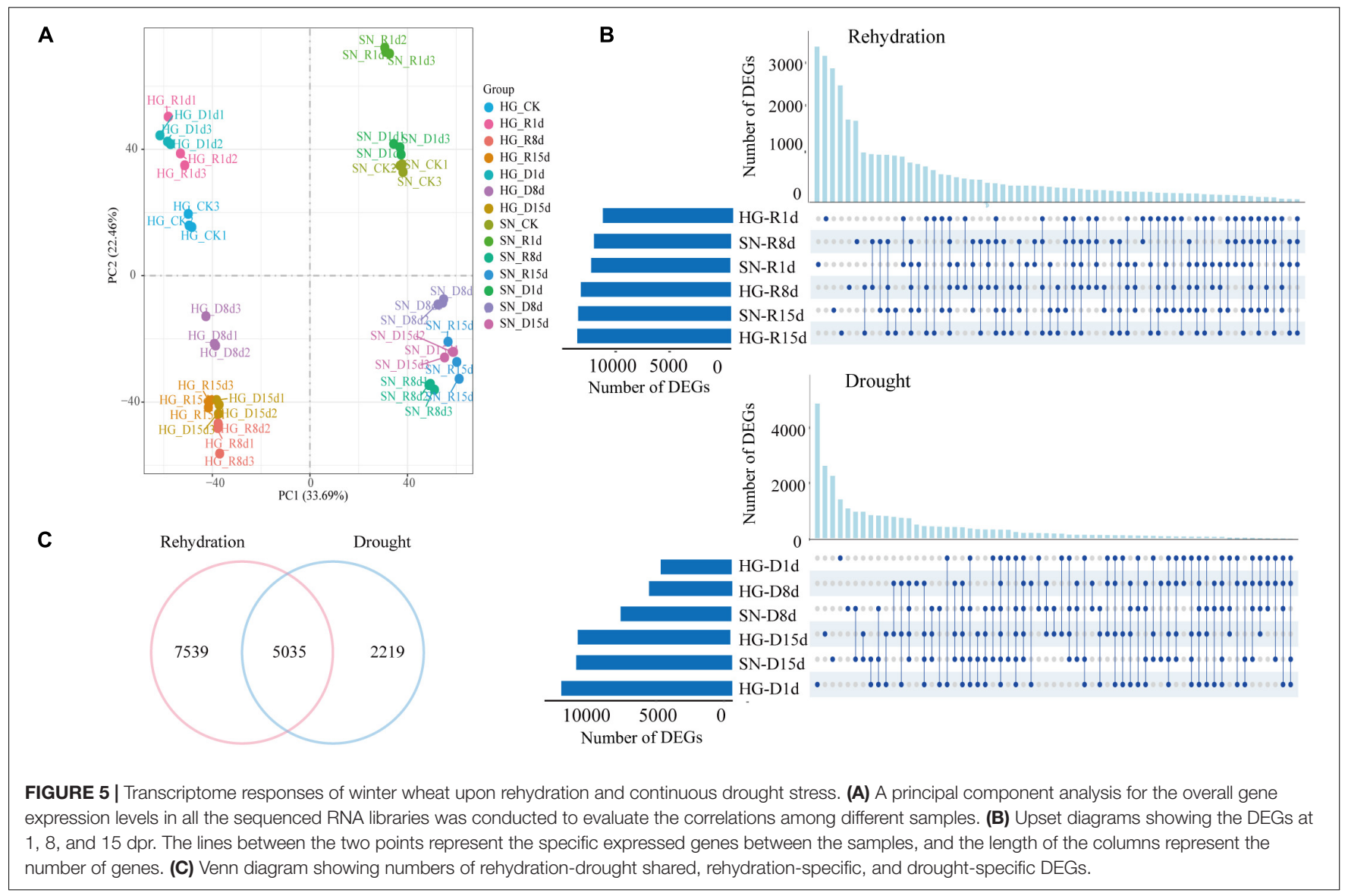


identified DEGs (approximately 40\%) encoded transcription factors (TFs), indicating a dynamic transcriptional change was triggered by the rehydration treatment (Supplementary Table 4).

\section{Rehydration Activated Biosynthetic Processes and Suppressed Stress-Response Pathway}

To further explore the core DEGs associated with rehydration or continuous drought stress, we checked the co-regulated DEGs between $\mathrm{HG}$ and $\mathrm{SN}$ at each time point. We identified 3,969, 6,964, and 6,230 co-regulated DEGs in both rehydrated HG and $\mathrm{SN}$ at 1, 8, and $15 \mathrm{dpr}$, respectively (Supplementary Figure 2). We combined all these genes and identified 12,574 non-redundant rehydration responsive DEGs. On the other hand, a total of 2,070, 2,492, and 5,087 co-regulated DEGs in both non-watered HG and SN were identified at 1, 8, and $15 \mathrm{dpr}$, respectively
(Figure 5B and Supplementary Figure 2). Combined from these genes, a total of 7,254 non-redundant drought responsive DEGs were identified. Interestingly, our further analysis revealed 5,035 shared genes between rehydration responsive DEGs and drought responsive DEGs (Figure 5C). Meanwhile, a total of 7,539 genes were designated as rehydration-specific DEGs and 2,219 genes as drought-specific DEGs.

A GO enrichment analysis was conducted on these rehydration-drought shared, rehydration-specific and drought-specific DEGs (Figure 6A). Rehydration-drought shared DEGs were enriched in carbohydrate biosynthetic process, carbohydrate catabolic process, and oxidoreduction coenzyme metabolic process. Rehydration-specific DEGs were enriched in chloroplast stroma, response to osmotic stress, and hormone-mediated signaling pathway. Drought-specific DEGs were involved in chloroplast stroma, carbohydrate biosynthetic process, and photosynthesis. More genes with GO
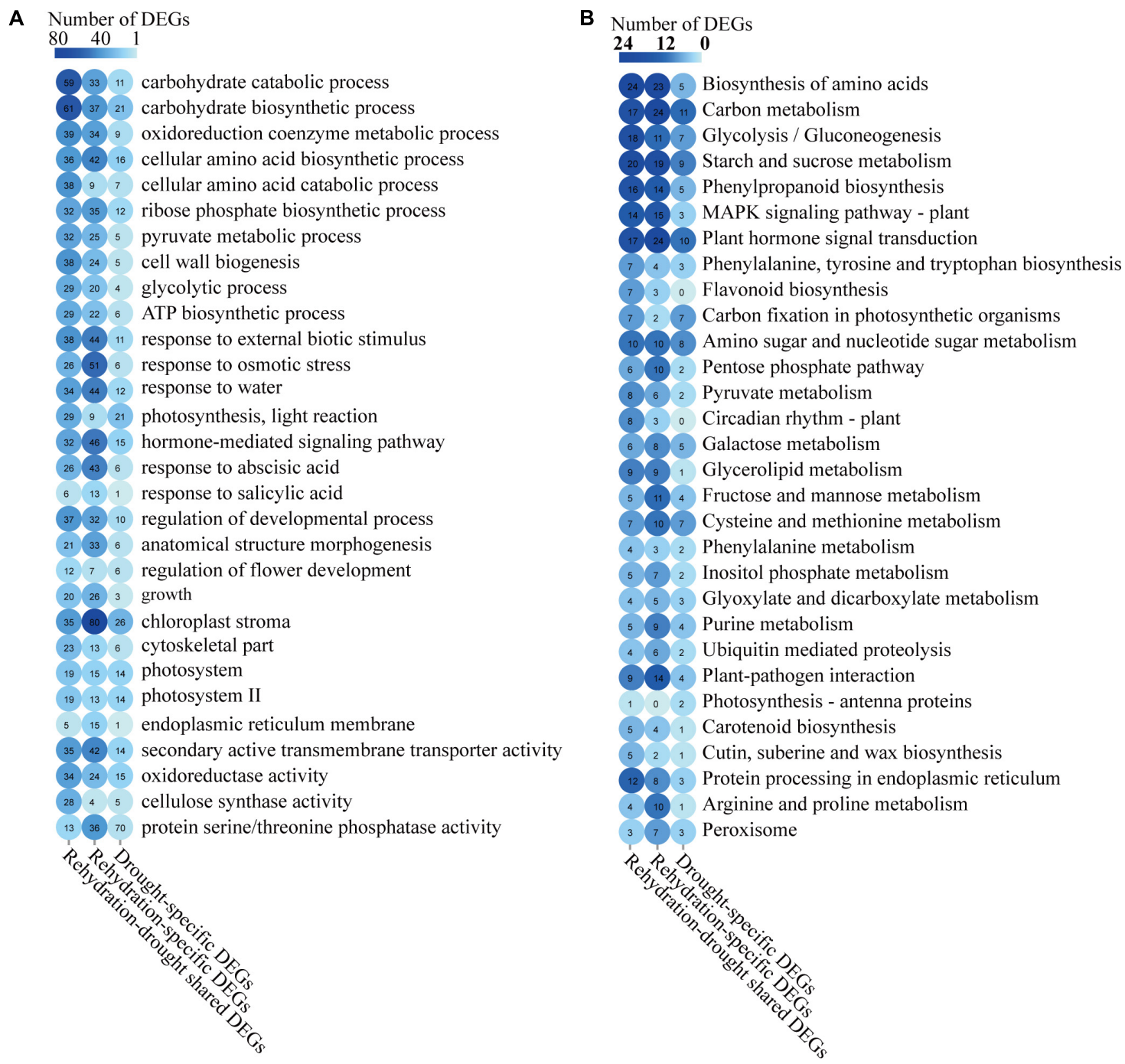

FIGURE 6 | GO and KEGG annotations of the rehydration-drought shared, rehydration-specific, and drought-specific DEGs. Enrichment of GO annotations (A) and KEGG pathways (B) in rehydration-drought shared, rehydration-specific, and drought-specific DEGs were summarized. 
annotations related to hormone-mediated signaling pathways, including genes response to $\mathrm{ABA}$ and $\mathrm{SA}$, were annotated in rehydration-specific DEGs than drought-specific DEGs.

KEGG annotations of these rehydration-drought shared, rehydration-specific and drought-specific DEGs were summarized to illustrate the regulatory pathways (Figure 6B). We found DEGs in all these three analysis groups were enriched in KEGG pathways of carbon metabolism, plant hormone signal transduction, and starch and sucrose metabolism. Compared with drought-specific DEGs, more genes in KEGG pathways of biosynthesis of amino acids, phenylpropanoid biosynthesis, and mitogen-activated protein kinase (MAPK) signaling pathway were annotated in rehydration-drought shared and rehydration-specific DEGs.

\section{Suppression of Genes Associated With Abscisic Acid Signaling Transduction Were Much Stronger in Hengguan35 Than in Shinong086 Upon Rehydration Treatment}

To reveal the molecular mechanisms controlling rehydration compensation in $\mathrm{HG}$, we analyzed the expression levels of genes enriched in the "plant hormone signal transduction" KEGG pathway (Figure 7). Two Triticum aestivum sucrose non-fermenting-1-related protein kinase 2 genes (TaSnRK2, TraesCS1A02G215900 and TraesCS2B02G521800) were significantly suppressed in $\mathrm{HG}$ at all three rehydration time points. A pyraclostrobin $(P Y R)$ or pyrabactin-resistancel-like $(P Y L)$ transcription factor gene (TraesCS2B02G105300) was upregulated at $8 \mathrm{dpr}$ in both $\mathrm{HG}$ and $\mathrm{SN}$, with a longer duration of induction in $\mathrm{HG}$ at $15 \mathrm{dpr}$. Three protein phosphatase type 2C genes (PP2C, TraesCS1B02G441400, TraesCS1B02G441400, and TraesCS5D02G188600) and two ABA-responsive element-binding factor genes (TraesCS6B02G364000 and TraesCS3D02G371900) were downregulated upon rehydration, with a more dramatic decrease in HG than SN. Moreover, the expression levels of several genes involved in the IAA, JA, and SA signaling pathways were also significantly altered.

\section{Hengguan35 Kept a More Balanced Expression of Genes Involved in ROS Pathway Than Shinong086}

We further analyzed DEGs involved in the ROS network to interpret their associations with oxidation reduction and
A

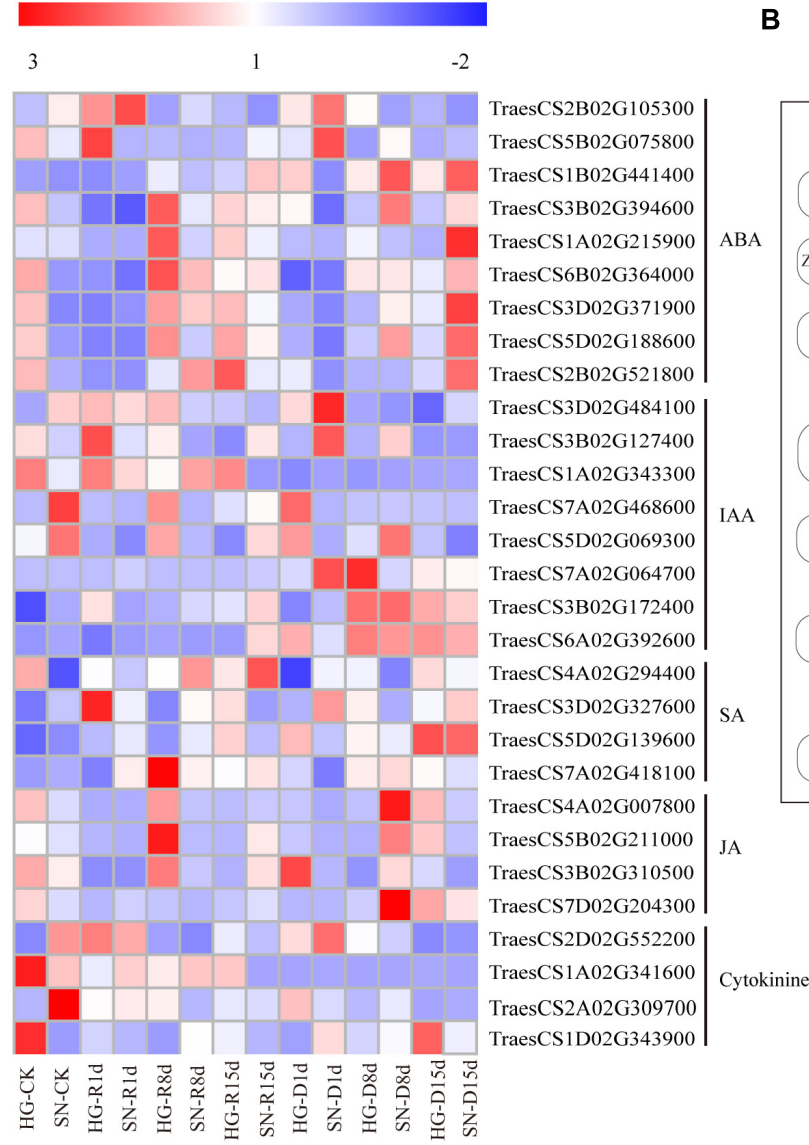

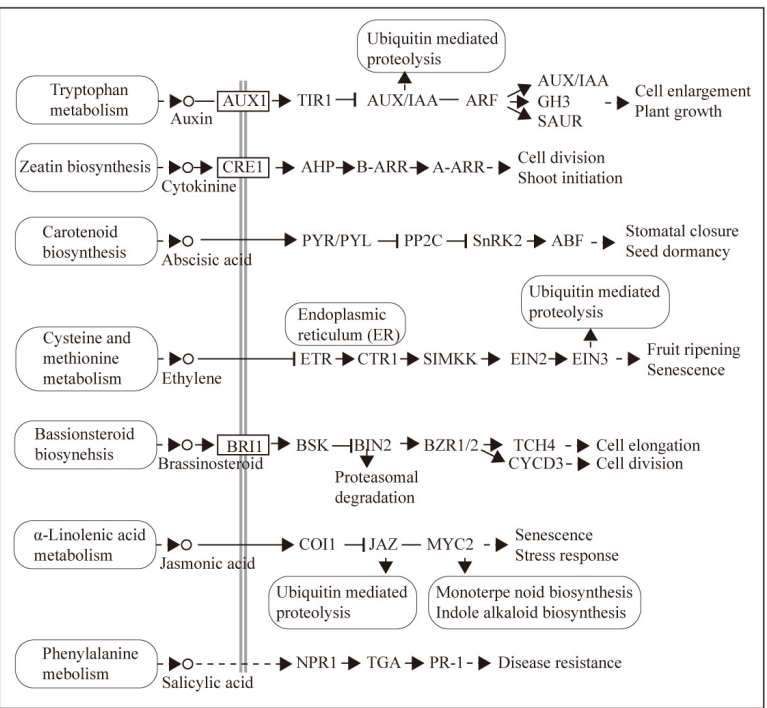

FIGURE 7 | Expression profiles of DEGs involved in plant hormone pathways. The expression profiles of transcripts (A) involved in hormone signal transduction pathways (B) were generated using their FPKM values. 
acclimation during rehydration compensation (Figure 8). The overall expression levels of three peroxisomal membrane protein 2 genes (PXMP2, TraesCS5B02G139100, TraesCS5A02G140800, and TraesCS5D02G151700) were downregulated upon rehydration, and had lower expression levels in HG compared with $\mathrm{SN}$. The mean platelet volume gene $(M p v$, TraesCS1B02G125400) controlling ROS metabolism had accumulated transcripts in $\mathrm{SN}$ but was suppressed in $\mathrm{HG}$. One of the Long-chain acyl-CoA synthetases genes (ACSL, TraesCS5D02G533700) was constantly downregulated

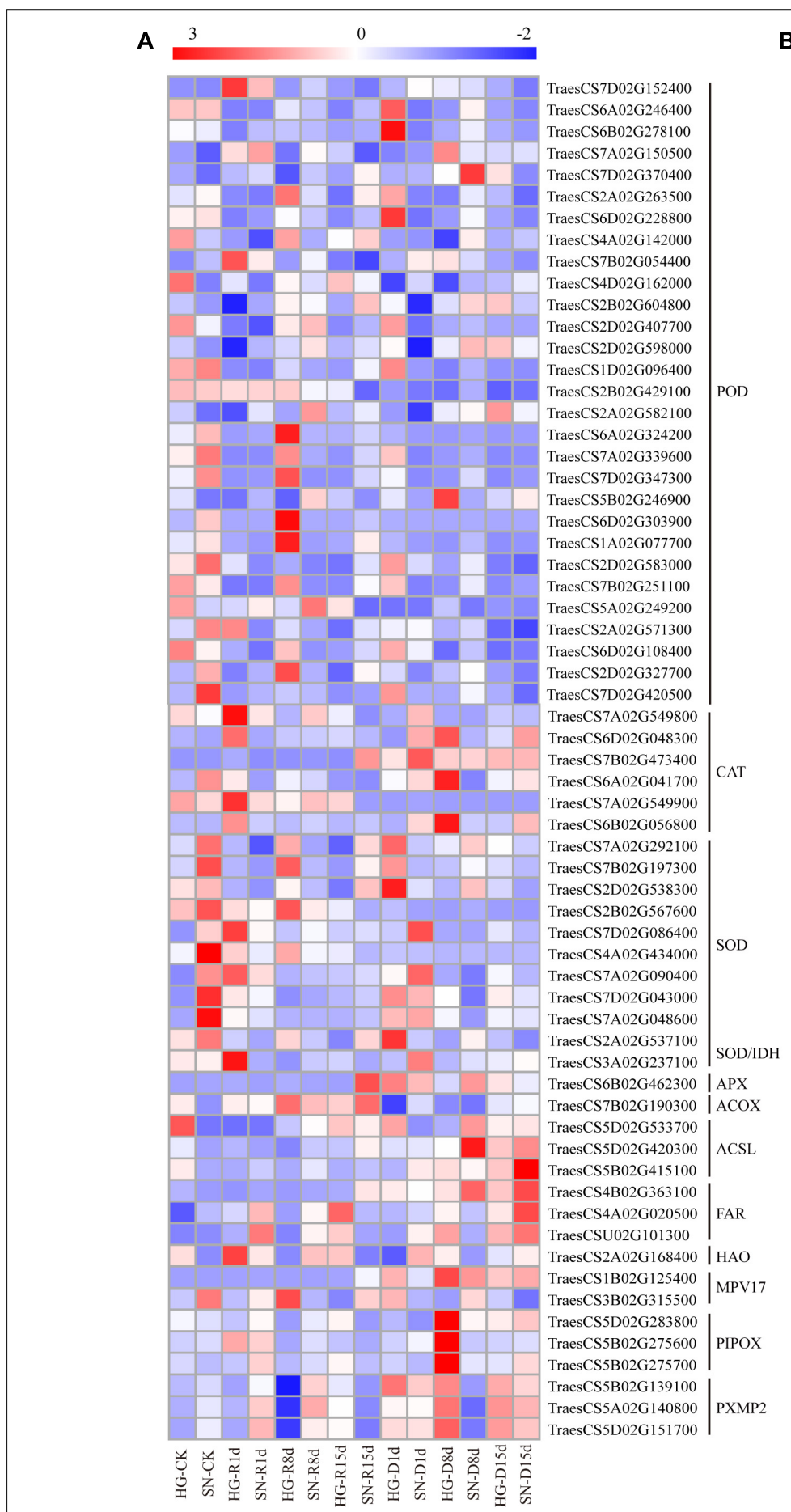

B

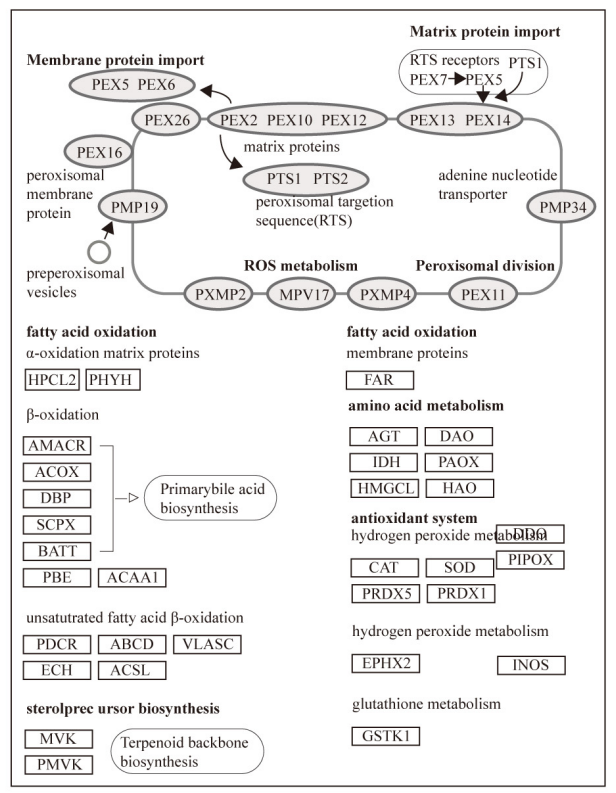

FIGURE 8 | Expression profiles of DEGs involved in ROS-related pathways. The expression levels of transcripts (A) in antioxidative and biosynthetic pathways (B) were generated using their FPKM values. 
in $H G$ after the rehydration treatment, whereas it was only downregulated at $8 \mathrm{dpr}$ in SN. Three SOD related genes (TraesCS4A02G434000, TraesCS7D02G043000, and TraesCS7A02G048600) were significantly upregulated at the early stage of rehydration. Most of the CAT related genes were induced at the late stage of rehydration. Interestingly, the overall expression level of one CAT related gene, TraesCS7B02G473400, in HG was significantly lower than that in $\mathrm{SN}$, whereas the other CAT related gene, TraesCS7A02G549900, showed an opposite expression pattern. Based on the expression profiles of these genes involved in the ROS network, we speculated that HG might utilize a more balanced ROS pathway to achieve a greater rehydration compensation level.

\section{Validation of Gene Expression by qRT-PCR}

To validate the expression patterns of genes determined by RNA-seq, Aquaporin TIP1-3 (TraesCS3A02G535300), leaf type ferredoxin-NADP and oxidoreductase (LFNR, TraesCS3A02G081300), Chlorophyll $a / b$ binding protein (CAB, TraesCS6A02G094400), alpha-D-phosphohexomutase superfamily (ADPS, TraesCS7A02G337000), Mitochondrial inner membrane protease ATP23 (MIMP, TraesCS2D02G340100), and Pyruvate dehydrogenase E1 component subunit alpha (PDHE1, TraesCS7D02G199900) were randomly selected from DEGs for a quantitative real-time PCR (qRT-PCR) analysis (Figure 9A). The wheat TaActin gene (GenBank accession AB181991.1) was used as an internal control. A high correlation $\left(R^{2}>0.9373\right)$ between overall expression levels from RNA-seq and qRT-PCR assays was observed (Figure 9B).

\section{DISCUSSION}

Winter wheat is susceptible to water scarcity, and high yields are normally associated with a sufficient water supply (Johnson et al., 2018). At present, wheat farmers have to consider both plant productivity and the costs of changing environmental factors (Xu et al., 2018; Yu et al., 2020). Monitoring plant morphological and biochemical changes upon rehydration after drought stress is important for characterizing drought-tolerant wheat genotypes (Jiang et al., 2020). In response to drought stress, plants adjust their flowering times to adapt to the vegetative growth stage (Shavrukov et al., 2017). Growth inhibition is an early response to drought stress (Kumar Jha et al., 2019). Rehydration at different growth stages can compensate for the decline in biomass caused by crop water deficit, especially after a moderate drought before the booting stage (Jiang et al., 2020). There is an obvious compensatory phenomenon in wheat plant height and leaf area (Li Y. et al., 2020), but there are rare reports of ear development on winter wheat after rehydration. In this work, we monitored the compensatory growth of two winter wheat varieties that have different drought resistance levels after rehydration (Figure 1). The promotion of plant growth and postponement of spike development were observed in winter wheat cultivars after rehydration. Moreover, the drought-tolerant cultivar showed a greater rehydration compensation than the drought-sensitive cultivar (Figure 2). For similar experiments in other crops, the elongation of young leaves in maize is extremely sensitive to the water supply and a rehydration treatment in the root medium immediately accelerates the growth of young leaves (Jiang et al., 2020). Rehydration compensation also delays the growth period to relieve the negative impacts of drought stress. It is considered a self-defense response of
A
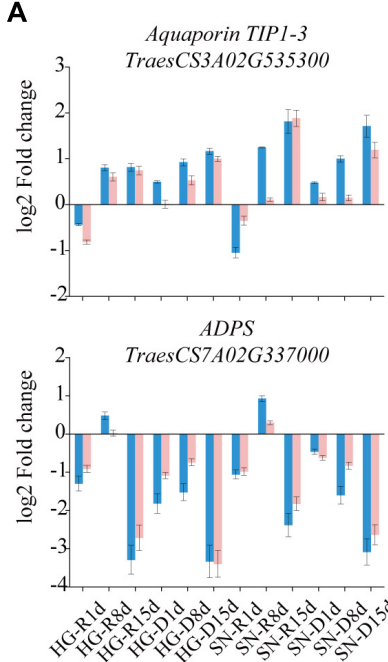
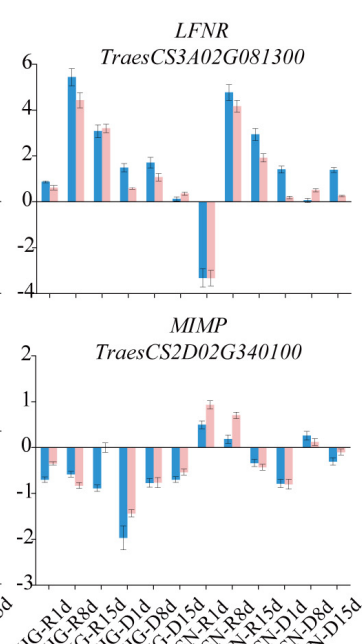

B

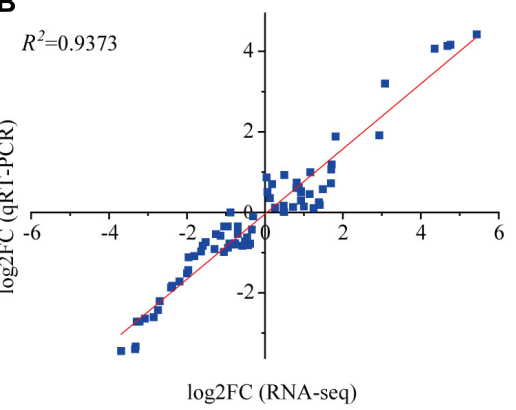

FIGURE 9 | The expression profiles of six randomly selected DEGs were validated by qRT-PCR assay. (A) The expression patterns of six randomly selected DEGs were generated using both qRT-PCR data and FPKM values. The relative transcript abundances were expressed relative to that of the internal reference TaActin following the $2^{-\Delta \Delta C t}$ method. FPKM values of these DEGs were collected from the transcriptome database. (B) Correlation analysis on the overall expression levels of six selected DEGs between RNA-seq and qRT-PCR. Log2FC values of RNA-seq data (x-axis) were plotted against log2FC values of qRT-PCR data (y-axis). R, rehydrated plants; D, plants under continuous drought stress. 
plants against short-term, periodic, or unpredictable drought (Mu et al., 2021). In addition, the compensation effect is largely dependent on the strength and duration of the stress (Rajala et al., 2009; Guo et al., 2014). For example, compared with the normally watered control, plant height increases and leaf area expands dramatically in soybean after rehydration (Dong et al., 2019).

Rehydration after drought stress induces complex biological processes in plant that require an energy balance between growth and stress avoidance (Zhu, 2016; Gupta et al., 2020). In plants, drought stress induces a wide range of responses, including increasing the oxidative damage in chloroplasts, inhibiting photosynthesis, suppressing metabolic responses, activating glycocatabolism, and changing cellular lipid compositions (Mittler and Blumwald, 2015; Yi et al., 2016; Li et al., 2019; Hrmova and Hussain, 2021; Waititu et al., 2021). ABA is the key phytohormone that mediates drought tolerance and rehydration compensation in plants (Saradadevi et al., 2017; Mega et al., 2019; Zhang et al., 2021). Upon rehydration, the levels of $\mathrm{ABA}$ and several other hormones, including IAA, GA, and cytokinin, first decline rapidly and then rise slowly, resulting in a "V"-shaped changing pattern (Dong et al., 2019). In the current investigation, we found dramatic decreases in the contents of $\mathrm{ABA}, \mathrm{GA}, \mathrm{JA}$, and $\mathrm{SA}$ upon rehydration (Figure 4). Genes encoding ABA receptors, including $P Y R$, $P Y L$, receptor component of $\mathrm{ABA}$ receptor $(R C A R)$ and $P P 2 C$, were differentially expressed after rehydration treatment as evidenced in the RNA-seq database (Figure 7). SnRK2 is a key kinase that phosphorylates many ABA downstream proteins (Lin et al., 2021). PP2C keeps SnRK2 inactive by blocking its catalytic cleft and de-phosphorylating its activation loop. However, the phosphorylation activity of PP2C is inhibited by ABA-PYL (Mosfeq-Ul Hasan et al., 2019; Li et al., 2021). Interactions between $\mathrm{PP} 2 \mathrm{C}$ and ABA-PYL eventually release SnRK2. In our transcriptome database, the TaPYR/PYL gene was upregulated, whereas both the TaPP2C and TaSnRK2 genes were downregulated after the rehydration treatment, indicating a suppression of the ABA pathway during rehydration compensation. Moreover, we noticed that expression levels of several ABA-signaling genes were much lower in $\mathrm{HG}$ than in $\mathrm{SN}$. Combined with evidence showing that the ABA content in $\mathrm{HG}$ was significantly lower than in $\mathrm{SN}$, we speculated that drought-tolerant wheat maintained a lower ABA content during drought stress and suppressed ABA signaling transduction upon rehydration.

Almost all abiotic stresses lead to the regulation of secondary oxidative stress stimulatory signals in a tissue-specific or even cell-specific manner (Chaves and Oliveira, 2004). Increases in the corresponding enzyme activities under stress conditions protects plant cells from oxidative damage (Chen et al., 2017; Zhang L. et al., 2020). To protect enzyme systems and membranes from lipid peroxidation, SOD, POD, and MDA activities increased at each growth stage after drought stress (Gill and Tuteja, 2010; Abid et al., 2018). In this study, contents of the soluble protein, soluble sugar, and Pro, as well as the activities of SOD, CAT, and POD, increased under drought stress, decreased rapidly upon rehydration and then increased gradually with the extension of rehydration (Figure 3). These dynamic changes were consistent with the results of previous investigations on corn, cotton, and other crops (Furlan et al., 2016; Voronin et al., 2019; Xu et al., 2019). Fluctuations in ROS are sensed by receptor proteins NADPH cytochrome $\mathrm{P} 450$ reductase (NPR) and heat stress response (HSF), redox-sensitive transcription factors, or phosphatases. Serine/threonine-protein kinases are involved in ROS induction, including the activation of mitogen-activated protein kinase, which further regulates the ROS-signaling network (Pitzschke and Hirt, 2009; Liu and He, 2017). The expression levels of genes in the ROS pathway are quantitatively influenced by the accumulation of ROS. In cotton, some transcription factor families, such as MYB, WRKY, AP2/ERF, and NAC, are involved in regulating plant responses to abiotic stress and ROSsignaling network (Mosfeq-Ul Hasan et al., 2019). In rice, myoinositol oxygenase (MIOX) family genes significantly improved the scavenging activity of ROS under drought stress (Duan et al., 2012). In this study, we observed association between the expression changes of ROS-related genes and rehydration treatment. Moreover, HG kept a more balanced expression of ROS-related genes than SN (Figure 8). Therefore, we speculate that rehydration of winter wheat induces the expression of ROS-related genes, reduces the contents of SOD, CAT, and MDA, and eventually diminishes the oxidative damage caused by drought stress.

\section{CONCLUSION}

In conclusion, we initially profiled the dynamic changes in physiological responses and gene expressions associated with rehydration in winter wheat under field conditions. Rehydration significantly promoted winter wheat growth and postponed spike development. A drought-tolerant variety showed a greater growth compensation than a drought-sensitive variety, which may be resulted from lower level of stressrelated phytohormones and activity of oxidoreductase. Genes involved in ABA signaling pathway and ROS balancing are playing crucial roles in the rehydration process. Our study provides new insights into the mechanism of rehydration compensation in crops and valuable gene resources for the genetic improvement of wheat.

\section{DATA AVAILABILITY STATEMENT}

The original contributions presented in the study are publicly available. This data can be found here: National Center for Biotechnology Information (NCBI) BioProject database under accession number PRJNA774165.

\section{AUTHOR CONTRIBUTIONS}

$\mathrm{WZ}$ conceived the project and set the scientific objectives. $\mathrm{XL}, \mathrm{XW}, \mathrm{PL}, \mathrm{XB}$, and $\mathrm{XH}$ contributed to the preparation of equipment and acquisition of data. XL, MY, and XW wrote the 
manuscript. All authors have read and approved the published version of the manuscript.

\section{FUNDING}

This research was funded by National Key R\&D Program of China (2017YFD0300906), the Foundation of State Key Laboratory of North China Crop Improvement and Regulation (NCCIR2020ZZ-16 and NCCIR2021ZZ-4), Modern

\section{REFERENCES}

Abid, M., Ali, S., Qi, L. K., Zahoor, R., Tian, Z., Jiang, D., et al. (2018). Physiological and biochemical changes during drought and recovery periods at tillering and jointing stages in wheat (Triticum aestivum L.). Sci. Rep 8:21441. doi: 10.1038/ s41598-018-21441-7

Ahmad, I., Khaliq, I., Mahmood, N., and Khan, N. (2015). Morphological and physiological criteria for drought tolerance at seedling stage in wheat. J. Anim. Plant Sci. 25, 1041-1048.

Anwar, A., Wang, K., Wang, J., Shi, L., Du, L., and Ye, X. (2021). Expression of Arabidopsis Ornithine Aminotransferase (AtOAT) encoded gene enhances multiple abiotic stress tolerances in wheat. Plant Cell Rep. 40, 1155-1170. doi: 10.1007/s00299-021-02699-0

Araya, A., Prasad, P. V. V., Gowda, P. H., Kisekka, I., and Foster, A. J. (2019). Yield and water productivity of winter wheat under various irrigation capacities. J. Am. Water Res. Assoc. 55, 24-37. doi: 10.1111/1752-1688.1 2721

Asada, Y. N. K. (1980). Hydrogen peroxide is scavenged by ascorbatespecific peroxidase in spinach chloroplasts. Plant Cell Physiol. 22, 867-880.

Ashraf, M. A., Rasheed, R., Hussain, I., Iqbal, M., Haider, M. Z., Parveen, S., et al. (2015). Hydrogen peroxide modulates antioxidant system and nutrient relation in maize (Zea mays L.) under water-deficit conditions. Arch. Agron. Soil Sci. 61, 507-523. doi: 10.1080/03650340.2014.938644

Baczek-Kwinta, R., Filek, W., Grzesiak, S., and Hura, T. (2006). The effect of soil drought and rehydration on growth and antioxidative activity in flag leaves of triticale. Biol. Plant. 50, 55-60. doi: 10.1007/s10535-005-0074-x

Bates, L. S., Waldren, R. P., and Teare, I. D. (1973). Rapid determination of free proline for water-stress studies. Plant Soil 39, 205-207. doi: 10.1007/bf00018060

Blum, A. (2017). Osmotic adjustment is a prime drought stress adaptive engine in support of plant production. Plant Cell Environ. 40, 4-10. doi: 10.1111/pce. 12800

Bramlage, Z. D. W. (1992). Modified thiobarbituric acid assay for measuring lipid oxidation in sugar-rich plant tissue extracts. J. Agricult. Food Chem. 40, 1566-1570. doi: 10.1021/jf00021a018

Caldeira, C. F., Bosio, M., Parent, B., Jeanguenin, L., Chaumont, F., and Tardieu, F. (2014). A hydraulic model is compatible with rapid changes in leaf elongation under fluctuating evaporative demand and soil water status. Plant Physiol. 164, 1718-1730. doi: 10.1104/pp.113.228379

Campion, E. M., Loughran, S. T., and Walls, D. (2011). Protein quantitation and analysis of purity. Methods Mol. Biol. 681, 229-258. doi: 10.1007/978-1-60761913-0_13

Chaves, M. M., and Oliveira, M. M. (2004). Mechanisms underlying plant resilience to water deficits: prospects for water-saving agriculture. J. Exp. Bot. 55, 23652384. doi: 10.1093/jxb/erh269

Chen, P., Jung, N. U., Giarola, V., and Bartels, D. (2020). The Dynamic Responses of Cell Walls in resurrection plants during dehydration and rehydration. Front. Plant Sci. 10:1698. doi: 10.3389/fpls.2019. 01698

Chen, Y. E., Cui, J. M., Su, Y. Q., Zhang, C. M., Ma, J., Zhang, Z. W., et al. (2017). Comparison of phosphorylation and assembly of photosystem complexes and redox homeostasis in two wheat cultivars with different drought resistance. Sci. Rep. 7:12718. doi: 10.1038/s41598-01713145-1 agricultural industrial technology system of Hebei Province (HBCT2018010205), and Provincial Natural Science Foundation of Hebei (C2021204008).

\section{SUPPLEMENTARY MATERIAL}

The Supplementary Material for this article can be found online at: https://www.frontiersin.org/articles/10.3389/fpls.2022. 823846/full\#supplementary-material

Dong, S., Jiang, Y., Dong, Y., Wang, L., Wang, W., Ma, Z., et al. (2019). A study on soybean responses to drought stress and rehydration. Saudi J. Biol. Sci. 26, 2006-2017. doi: 10.1016/j.sjbs.2019.08.005

Du, Y., Zhao, Q., Chen, L., Yao, X., and Xie, F. (2020). Effect of drought stress at reproductive stages on growth and nitrogen metabolism in soybean. Agronomy 10:302.

Duan, J., Zhang, M., Zhang, H., Xiong, H., and Li, Z. (2012). OsMIOX, amyoinositol oxygenase gene, improves drought tolerance through scavenging of reactive oxygen species in rice (Oryza sativa L.). Plant Sci. 196, 143-151. doi: 10.1016/j.plantsci.2012.08.003

Furlan, A., Bianucci, E., Tordable, M. D., Kleinert, A., Valentine, A., and Castro, S. (2016). Dynamic responses of photosynthesis and the antioxidant system during a drought and rehydration cycle in peanut plants. Funct. Plant Biol. 43, 337-345. doi: 10.1071/FP15206

Giannopolitis, C. N., and Ries, S. K. (1977). Superoxide dismutases: I. Occurrence in higher plants. Plant Physiol. 59, 309-314. doi: 10.1104/pp.59.2.309

Gill, S. S., and Tuteja, N. (2010). Reactive oxygen species and antioxidant machinery in abiotic stress tolerance in crop plants. Plant Physiol. Biochem. 48, 909-930. doi: 10.1016/j.plaphy.2010.08.016

Guo, Z., Yu, Z., Wang, D., Shi, Y., and Zhang, Y. (2014). Photosynthesis and winter wheat yield responses to supplemental irrigation based on measurement of water content in various soil layers. Field Crops Res. 166, 102-111. doi: 10.1016/j.fcr.2014.06.004

Gupta, A., Rico-Medina, A., and Caño, D. (2020). The physiology of plant responses to drought. Science 368, 266-269. doi: 10.1126/science.aaz7614

Han, L. B., Song, G. L., and Zhang, X. (2008). Preliminary observations on physiological responses of three turfgrass species to traffic stress. Horttechnology 18, 139-143. doi: 10.21273/horttech.18.1.139

He, J., Duan, Y., Hua, D., Fan, G., Wang, L., Liu, Y., et al. (2012). DEXH box RNA helicase-mediated mitochondrial reactive oxygen species production in Arabidopsis mediates crosstalk between abscisic acid and auxin signaling. Plant Cell 24, 1815-1833. doi: 10.1105/tpc.112.098707

Hrmova, M., and Hussain, S. S. (2021). Plant transcription factors involved in drought and associated stresses. Int. J. Mol. Sci. 22:5662. doi: 10.3390/ ijms22115662

Huang, J., Ridoutt, B. G., Thorp, K. R., Wang, X., Lan, K., Liao, J., et al. (2019). Water-scarcity footprints and water productivities indicate unsustainable wheat production in China. Agricult. Water Manag. 224:5744.

Husen, A., Iqbal, M., and Aref, I. M. (2014). Growth, water status, and leaf characteristics of Brassica carinata under drought and rehydration conditions. Brazil. J. Bot. 37, 217-227. doi: 10.1007/s40415-014-0066-1

Jiang, T., Dou, Z., Liu, J., Gao, Y., Malone, R. W., Chen, S., et al. (2020). Simulating the influences of soil water stress on leaf expansion and senescence of winter wheat. Agricult. Forest Meteorol. 291:108061. doi: 10.1016/j.agrformet.2020. 108061

Johnson, K. M., Jordan, G. J., and Brodribb, T. J. (2018). Wheat leaves embolized by water stress do not recover function upon rewatering. Plant Cell Environ. 41, 2704-2714. doi: 10.1111/pce.13397

Khadka, K., Earl, H. J., Raizada, M. N., and Navabi, A. (2020). A physiomorphological trait-based approach for breeding drought tolerant wheat. Front. Plant Sci. 11:715. doi: 10.3389/fpls.2020.00715

Kim, D., Langmead, B., and Salzberg, S. L. (2015). HISAT: a fast spliced aligner with low memory requirements. Nat. Methods 12, 357-360. doi: 10.1038/nmeth. 3317 
Koffler, B. E., Luschin-Ebengreuth, N., Stabentheiner, E., Mueller, M., and Zechmann, B. (2014). Compartment specific response of antioxidants to drought stress in Arabidopsis. Plant Sci. 227, 133-144. doi: 10.1016/j.plantsci. 2014.08.002

Kong, X., Zhang, X., Lal, R., Zhang, F., Chen, X., Niu, Z., et al. (2016). Groundwater depletion by agricultural tntensification in China's $\mathrm{HHH}$ plains, since 1980s. Adv. Agron. 135, 59-106.

Kumar Jha, S., Ramatshaba, T. S., Wang, G., Liang, Y., Liu, H., Gao, Y., et al. (2019). Response of growth, yield and water use efficiency of winter wheat to different irrigation methods and scheduling in North China Plain. Agricult. Water Manag. 217, 292-302. doi: 10.1016/j.agwat.2019.03.011

Li, D., Batchelor, W. D., Zhang, D., et al. (2020). Analysis of melatonin regulation of germination and antioxidant metabolism in different wheat cultivars under polyethylene glycol stress[J]. PLoS One 15:e237536. doi: 10.1371/journal.pone. 0237536

Li, J., Li, Y., Yin, Z., Jiang, J., Zhang, M., Guo, X., et al. (2017). OsASR5 enhances drought tolerance through a stomatal closure pathway associated with ABA and $\mathrm{H}_{2} \mathrm{O}_{2}$ signalling in rice. Plant Biotechnol. J. 15, 183-196. doi: 10.1111/pbi.1 2601

Li, L., Li, B., Zhu, S., Wang, L., Song, L., Chen, J., et al. (2021). TMK4 receptor kinase negatively modulates ABA signaling by phosphorylating ABI2 and enhancing its activity. J. Integ. Plant Biol. 63, 1161-1178. doi: 10.1111/jipb. 13096

Li, X. R., Tang, Y., Zhou, C. J., Zhang, L. X., and Lv, J. Y. (2020). A wheat WRKY transcription factor TaWRKY46 enhances tolerance to osmotic stress in transgenic Arabidopsis plants. Int. J. Mol. Sci. 21:1321. doi: 10.3390/ ijms21041321

Li, Y., Li, H., Zhang, S., and Wang, Y. (2020). Ear photosynthetic anatomy effect on wheat yield and water use efficiency. Agron. J. 112, 1778-1793. doi: 10.1002/ agj2.20154

Li, Y., Son, H., Zhou, L., Xu, Z., and Zhou, G. (2019). Tracking chlorophyll fluorescence as an indicator of drought and rewatering across the entire leaf lifespan in a maize field. Agricult. Water Manag. 211, 190-201. doi: 10.1016/j. agwat.2018.09.050

Lin, Z., Li, Y., Wang, Y., Liu, X., Ma, L., Zhang, Z., et al. (2021). Initiation and amplification of SnRK2 activation in abscisic acid signaling. Nat. Commun. 12, 2456-2456. doi: 10.1038/s41467-021-22812-x

Liu, Y., and He, C. (2017). A review of redox signaling and the control of MAP kinase pathway in plants. Redox Biol. 11, 192-204. doi: 10.1016/j.redox.2016. 12.009

Livak, K. J., and Schmittgen, T. D. (2001). Analysis of relative gene expression data using real-time quantitative PCR and the 2(T)(-Delta Delta C) method. Methods 25, 402-408. doi: 10.1006/meth.2001.1262

Lopes, M. S., Reynolds, M. P., Jalal-Kamali, M. R., Moussa, M., Feltaous, Y., Tahir, I. S. A., et al. (2012). The yield correlations of selectable physiological traits in a population of advanced spring wheat lines grown in warm and drought environments. Field Crops Res. 128, 129-136.

Mega, R., Abe, F., Kim, J. S., Tsuboi, Y., Tanaka, K., Kobayashi, H., et al. (2019). Tuning water-use efficiency and drought tolerance in wheat using abscisic acid receptors. Nat. Plants 5, 153-159. doi: 10.1038/s41477-019-0361-8

Miller, G. L. (1959). Use of dinitrosalicylic acid reagent for determination of reducing sugar. Analy. Chem. 31, 426-428. doi: 10.1021/ac6014 $7 \mathrm{a} 030$

Mittler, R., and Blumwald, E. (2015). The roles of ROS and ABA in systemic acquired acclimation. Plant Cell 27, 64-70. doi: 10.1105/tpc.114.133090

Mosfeq-Ul Hasan, M., Ma, F. L., Islam, F., Sajid, M., Prodhan, Z. H., Li, F., et al. (2019). Comparative transcriptomic analysis of biological process and key pathway in three cotton (Gossypium spp.) species under drought stress. Int. J. Mol. Sci. 20:76. doi: 10.3390/ijms20092076

Mu, Q., Cai, H., Sun, S., Wen, S., Xu, J., Dong, M., et al. (2021). The physiological response of winter wheat under short-term drought conditions and the sensitivity of different indices to soil water changes. Agricult. Water Manag. 243:106475.

Pitzschke, A., and Hirt, H. (2009). Disentangling the complexity of mitogenactivated protein kinases and reactive oxygen species signaling. Plant Physiol. 149, 606-615. doi: 10.1104/pp.108.131557

Qiu, J. R., Xiang, X. Y., Wang, J. T., Xu, W. X., Chen, J., Xiao, Y., et al. (2020). MfPIF1 of resurrection plant Myrothamnus flabellifolia plays a positive regulatory role in responding to drought and salinity stresses in Arabidopsis. Int. J. Mol. Sci. 21:3011. doi: 10.3390/ijms21083011

Rajala, A., Hakala, K., Makela, P., Muurinen, S., and Peltonen-Sainio, P. (2009). Spring wheat response to timing of water deficit through sink and grain filling capacity. Field Crops Res. 114, 263-271. doi: 10.1016/j.fcr.2009.08.007

Robinson, M. D., Mccarthy, D. J., and Smyth, G. K. (2010). edgeR: a Bioconductor package for differential expression analysis of digital gene expression data. Bioinformatics 26, 139-140. doi: 10.1093/bioinformatics/btp616

Rong, W., Luo, M., Shan, T., Wei, X., Du, L., Xu, H., et al. (2016). A Wheat cinnamyl alcohol dehydrogenase TaCAD12 contributes to host resistance to the sharp eyespot disease. Front. Plant Sci. 7:1723. doi: 10.3389/fpls.2016.01723

Sajjad, M., Wei, X., Liu, L., Li, F., and Ge, X. (2021). Transcriptome analysis revealed GhWOX4 intercedes myriad regulatory pathways to modulate drought tolerance and vascular growth in cotton. Int. J. Mol. Sci. 22:898. doi: 10.3390/ ijms22020898

Saradadevi, R., Palta, J. A., and Siddique, K. H. M. (2017). ABA-mediated stomatal response in regulating water use during the development of terminal drought in wheat. Front. Plant Sci. 8:1251. doi: 10.3389/fpls.2017.01251

Shavrukov, Y., Kurishbayev, A., Jatayev, S., Shvidchenko, V., Zotova, L., Koekemoer, F., et al. (2017). Early flowering as a drought escape mechanism in plants: How can it aid wheat production? Front. Plant Sci. 8:1950. doi: 10.3389/fpls.2017. 01950

Shimizu, M. K. S. (1987). Chlorophyll metabolism in higher plants. VII. Chlorophyll degradation in senescing tobacco leaves; phenolic-dependent peroxidative degradation. Canad. J. Bot. 65, 729-735. doi: 10.1139/b87-097

Song, Q., Liu, C., Bachir, D. G., Chen, L., and Hu, Y. G. (2017). Drought resistance of new synthetic hexaploid wheat accessions evaluated by multiple traits and antioxidant enzyme activity. Field Crops Res. 210, 91-103. doi: 10.1016/j.fcr. 2017.05.028

Trujillo, I., Rivas, M., and Castrillo, M. (2013). Leaf recovery responses during rehydration after water deficit in two bean (Phaseolus vulgaris L.) cultivars. J. Plant Interact. 8, 360-369.

Truskina, J., Han, J., Chrysanthou, E., Galvan-Ampudia, C. S., Laine, S., Brunoud, G., et al. (2021). A network of transcriptional repressors modulates auxin responses. Nature 589, 116-119.

Voronin, P. Y., Maevskaya, S. N., and Nikolaeva, M. K. (2019). Physiological and molecular responses of maize (Zea mays L.) plants to drought and rehydration. Photosynthetica 57, 850-856.

Waititu, J. K., Zhang, X., Chen, T., Zhang, C., Zhao, Y., and Wang, H. (2021). Transcriptome analysis of tolerant and susceptible maize genotypes reveals novel insights about the molecular mechanisms underlying drought responses in leaves. Int. J. Mol. Sci. 22:6980. doi: 10.3390/ijms22136980

Wang, N., Gao, J., and Zhang, S. Q. (2017). Overcompensation or limitation to photosynthesis and root hydraulic conductance altered by rehydration in seedlings of sorghum and maize. Crop J. 5, 337-344.

Wang, T. Z., Zhang, J. L., Tian, Q. Y., Zhao, M. G., and Zhang, W. H. (2013). A Medicago truncatula EF-Hand family gene, MtCaMP1, is involved in drought and salt stress tolerance. PLoS One 8:e58952. doi: 10.1371/journal. pone.0058952

Wang, X., Li, L., Ding, Y., Xu, J., Wang, Y., Zhu, Y., et al. (2021). Adaptation of winter wheat varieties and irrigation patterns under future climate change conditions in Northern China. Agricult. Water Manag. 243:6409.

Xu, X., Zhang, M., Li, J., Liu, Z., Zhao, Z., Zhang, Y., et al. (2018). Improving water use efficiency and grain yield of winter wheat by optimizing irrigations in the North China Plain. Field Crops Res. 221, 219-227.

Xu, Y. C., Magwanga, R. O., Cai, X. Y., Zhou, Z. L., Wang, X. X., Wang, Y. H., et al. (2019). Deep transcriptome analysis reveals reactive oxygen species (ROS) network evolution, response to abiotic stress, and regulation of fiber development in cotton. Int. J. Mol. Sci. 20:1863. doi: 10.3390/ijms20081863

Ye, J., Wang, S., Deng, X., Yin, L., Xiong, B., and Wang, X. (2016). Melatonin increased maize (Zea mays L.) seedling drought tolerance by alleviating drought-induced photosynthetic inhibition and oxidative damage. Acta Physiol. Plantarum 38:45.

Yi, F., Gu, W., Chen, J., Song, N., Gao, X., Zhang, X., et al. (2019). High temporalresolution transcriptome landscape of early maize seed development. Plant cell 31, 974-992. doi: 10.1105/tpc.18.00961

Yi, X. P., Zhang, Y. L., Yao, H. S., Luo, H. H., Gou, L., Chow, W. S., et al. (2016). Different strategies of acclimation of photosynthesis, electron transport and 
antioxidative activity in leaves of two cotton species to water deficit. Funct. Plant Biol. 43, 448-460. doi: 10.1071/FP15247

Yu, L., Zhao, X., Gao, X., and Siddique, K. H. M. (2020). Improving/maintaining water-use efficiency and yield of wheat by deficit irrigation: A global metaanalysis. Agricult. Water Manag. 228:105906. doi: 10.1016/j.agwat.2019.105906

Zhang, L., Lei, D., Deng, X., Li, F., Ji, H., and Yang, S. (2020). Cytosolic glyceraldehyde-3-phosphate dehydrogenase 2/5/6 increase drought tolerance via stomatal movement and reactive oxygen species scavenging in wheat. Plant Cell Environ. 43, 836-853. doi: 10.1111/pce.13710

Zhang, Q., Yuan, W., Wang, Q., Cao, Y., Xu, F., Dodd, I. C., et al. (2021). ABA regulation of root growth during soil drying and recovery can involve auxin response. Plant Cell Environ. 2021:14137. doi: 10.1111/pce.14137

Zhang, S. C., Yang, R., Huo, Y. Q., Liu, S. S., Yang, G. D., Huang, J. G., et al. (2018). Expression of cotton PLATZ1 in transgenic Arabidopsis reduces sensitivity to osmotic and salt stress for germination and seedling establishment associated with modification of the abscisic acid, gibberellin, and ethylene signalling pathways. BMC Plant Biol. 18:218. doi: 10.1186/s12870-018-1416-0

Zhang, Z., Zhu, L., Song, A., Wang, H., Chen, S., Jiang, J., et al. (2020). Chrysanthemum (Chrysanthemum morifolium) CmICE2 conferred freezing tolerance in Arabidopsis. Plant Physiol. Biochem. 146, 31-41. doi: 10.1016/j. plaphy.2019.10.041

Zhao, J., Han, T., Wang, C., Jia, H., Worqlul, A. W., Norelli, N., et al. (2020). Optimizing irrigation strategies to synchronously improve the yield and water productivity of winter wheat under interannual precipitation variability in the North China Plain. Agricult. Water Manag. 240: 106298.
Zheng, J., Fu, J., Gou, M., Huai, J., Liu, Y., Jian, M., et al. (2010). Genome-wide transcriptome analysis of two maize inbred lines under drought stress. Plant Mol. Biol. 72, 407-421. doi: 10.1007/s11103-009-9579-6

Zhong, Y., Gao, C., Dong, Z., Chen, N., and Wang, M. (2013). Determination of five endogenous hormones in wheat by high performance liquid chromatography. Chinese J. Chromatograp. 31, 800-803. doi: 10.3724/sp.j.1123.2013.0 1011

Zhu, J. K. (2016). Abiotic stress signaling and responses in plants. Cell 167, 313-324.

Conflict of Interest: The authors declare that the research was conducted in the absence of any commercial or financial relationships that could be construed as a potential conflict of interest.

Publisher's Note: All claims expressed in this article are solely those of the authors and do not necessarily represent those of their affiliated organizations, or those of the publisher, the editors and the reviewers. Any product that may be evaluated in this article, or claim that may be made by its manufacturer, is not guaranteed or endorsed by the publisher.

Copyright $\odot 2022$ Liu, Wang, Liu, Bao, Hou, Yang and Zhen. This is an open-access article distributed under the terms of the Creative Commons Attribution License (CC BY). The use, distribution or reproduction in other forums is permitted, provided the original author(s) and the copyright owner(s) are credited and that the original publication in this journal is cited, in accordance with accepted academic practice. No use, distribution or reproduction is permitted which does not comply with these terms. 